\title{
Microstructures in phase-inversion membranes. Part 1. Formation of macrovoids*
}

\author{
C.A. Smolders, A J. Reuvers, R.M. Boom and I.M. Wienk \\ Department of Chemical Technology, Unwersity of Twente, $P$ O Box 217, 7500 AE Enschede (The Netherlands)
}

(Received July 31, 1991, accepted in revised form June 11, 1992)

\begin{abstract}
A new mechanism for the formation of macrovolds in phase-inversion membranes is proposed It is based on the observed difference in type of demixing of a thin film of a polymer solution when immersed in a nonsolvent bath delayed or instantaneous demixing The explanation for macrovold formation assumes local conditions of delayed demixing in front of a certain layer of nuclei already formed, due to a change in interfacial compositions at the advancing coagulation front, as compared to the onginal composition at the interface film-bath Effects of variations in membrane formation conditions which can be calculated using the model of diffusive mass transport in thin films of polymeric solutions in combination with phase separation in phase-inversion membranes, completely support the mechanism as proposed

Keywords membrane preparation and structure, theory, thermodynamics, macrovolds, phase-inversion membranes
\end{abstract}

\section{Introduction}

The preparation of asymmetric membranes by the phase-inversion process is a standard technique nowadays. A thin film of a homogeneous polymer solution is contacted with a second liquid which is a nonsolvent for the polymer but which is miscible in all proportions with the polymer solvent. Exchange of solvent and nonsolvent across the interface introduces phase separation in the polymer film, which can

Correspondence to C A Smolders, Department of Chemical Technology, University of Twente, P O Box 217, 7500 AE Enschede, The Netherlands

*Paper presented at the Int Symp on "Progress in Membrane Science and Technology", Enschede, The Netherlands, June 25-28, 1992 lead to a variety of characteristic asymmetric or symmetric structures. Examples are:

- a dense toplayer is formed, supported by a layer containıng closed cell pores or pores of an open interconnected type. In optimized form these membranes can separate low molecular weight mixed components by reverse osmosis, pervaporation or gas separation;

- a thin toplayer is formed with a typical structure of closely packed polymeric spheres, a socalled nodular structure (nodule size 20-100 $\mathrm{nm}$ ), supported by a very open porous layer, often containing large elongated voids, called macrovoids. This type of membrane ca be optimized to separate dissolved molecules of colloidal size from low molecular weight solutes by ultrafiltration, or they can be employed as sup- 
port layer for the preparation of composite membranes,

- an interconnected pore structure is formed with little variation in pore size $(0.1-1 \mu \mathrm{m})$ over the membrane thickness Optımized membranes of this type can be used to separate suspended material (e g bacteria) from fluids by microfiltration.

The formation of characteristic structures in phase-inversion membranes has been described by a model for diffusive mass transport in thin films of polymeric solutions and its effect on phase separation by liquid-liquid demixing [1-3] The formation of two types of these structures, both occurring in ultrafiltration membranes have not been satisfactorly explained nodular structures and macrovords While we will give special attention to nodule formation in Part 2 of this study [4] we will concentrate on formation of macrovoids in this part.

The mentioned model assumes the existence of a constant composition at the interface between polymer solution and coagulation bath. It has been shown by Tsay and McHugh [5] that this assumption is not correct By not simplifyıng the diffusion equations to ordinary differential equations but by calculating the true partial differential equations, they eliminated the assumption of constant composition at the interface between coagulation bath and polymer film [2]. Therefore their mass transfer model could more adequately predict the delay time of demixing However, for the understanding of the formation of macrovoids, such a complicated and calculation-intensive model is not needed, as will be shown in this paper We will therefore restrict ourselves to the Reuvers model

Experimentally it has been shown that the formation of macrovoids in membranes prepared from a polymer-solvent-nonsolvent system can be suppressed or even elıminated by - choosing a solvent-nonsolvent pair with a low tendency of mixing with each other [6,7], - increasing the polymer concentration in the casting solution or allowing the solvent to evaporate from the solution film before immersion into the nonsolvent bath $[7,8]$, - addition of solvent to the coagulation bath $[8,9]$

It is generally concluded from these observations that macrovoid formation occurs under rapid precipitation conditions and that void formation can be suppressed by increasing the viscosity of the casting solution [6-8,10-12].

In this paper we will first summarize the main conclusions from the Reuvers model for mass transfer [3] in combination with phase separation during membrane formation Then we derive from literature data some empirical rules for the appearance of macrovoids in membranes and we will present a new model [3a] for the formation of macrovoids which is in accordance with practice

\section{Theoretical background on the formation of phase inversion membranes}

From the model given by Reuvers et al [3] for membrane formation a new mechanism for macrovoid formation can be formulated. In order to treat this properly a clear notion is required of two distinctly different ways in which liquid-liquid demixing in the immersed polymeric solution can be effectuated, characterized by the moment of onset of phase separation. This moment either coincides with the very moment at which the solution film is contacted with the nonsolvent bath (1 e. instantaneous demixing), or it is delayed for a certain period, during which the changes in composition in the film will not be disrupted by phase separation (delayed demixing). For a detalled treatment of the model for combined diffusion and phase separation in membrane formation the reader is referred to earlier publications 
[3b,c] Analogous equations for a quaternary system are given in Part 2 [4]

A brief summary of the most important points and some calculated results character1zing delayed and instantaneous precipitation will be given here:

- Equilibrium phase diagrams in ternary poly$\mathrm{mer} /$ solvent/nonsolvent systems are calculated using Flory-Huggins formalism and measured data for the required interaction parameters [13]. These phase diagrams and the thermodynamic expressions for the chemical potentials in the system are necessary to properly describe the diffusion process upon $1 \mathrm{~m}$ mersion in a nonsolvent bath (boundary conditions, metastable/unstable compositions etc ).

- Ternary diffusion equations are formulated relating chemical potential gradients in the polymer film with frictional coefficients between the various components. The frictional coefficients required are obtained (with reasonable underlyıng assumptions for converting ternary parameters into binary ones [3c] ) from binary diffusion and sedimentation experıments Also for the binary diffusion process in the coagulation bath equations and required coefficients are obtained Since the model is based on the theory of irreversible thermodynamics we assume that everywhere in the system local equilibrium is present, i.e. there are no discontinuities in the chemical potentials of the components This is therefore also assumed on the interface

- The model calculations for diffusion finally result in so-called composition paths in the phase diagrams (or concentration profiles in the immersed film), up to the moment that demixing in the film sets in. As long as the interface composition can be considered constant, the composition path can be interpreted in two different ways The composition path is the complete range of compositions present at a certain fixed time It is however also the range of compositions that one single volume element assumes during all times of the immersion process

Two characteristic results of these calculations are reproduced here, giving an example of delayed ( $F_{1} g$ 1) and instantaneous onset of demixing (F1g 2), respectıvely The parameters

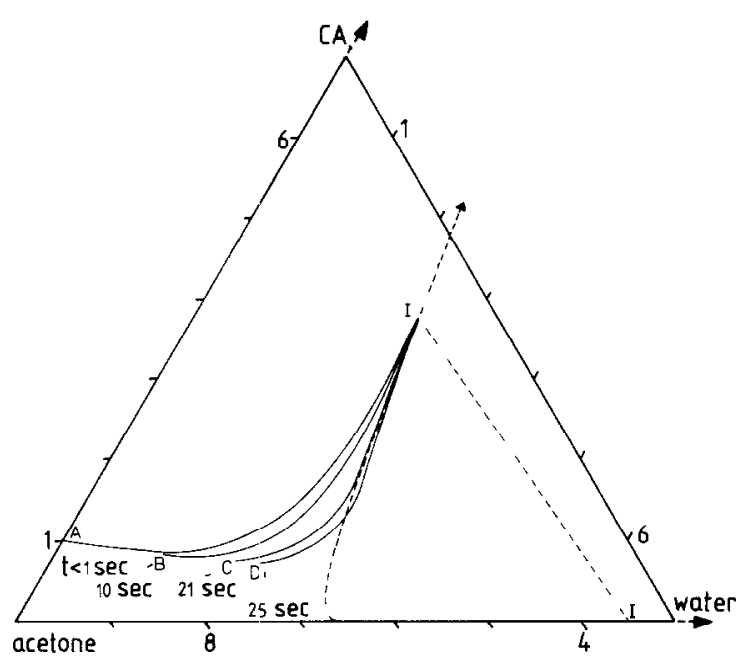

Fig 1 Approximated change of the composition path at different immersion times for a $10 \mathrm{vol} \% \mathrm{CA}$ solution $\mathrm{im}$ mersed into a pure water bath, initial film thickness 200 $\mu \mathrm{m}$

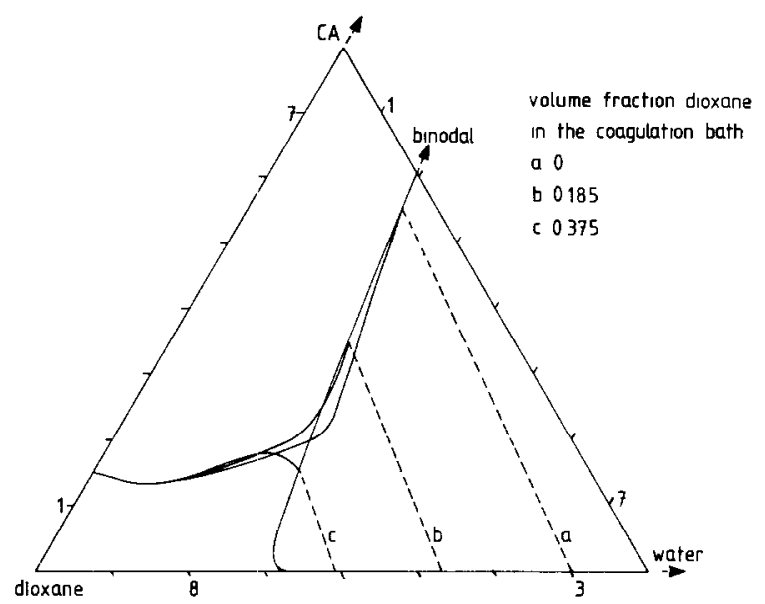

Fig 2 Calculated initial composition paths for $15 \mathrm{vol} \%$ CA-dioxane solutions immersed into a coagulation bath with a varying initial composition 
used in the calculations are given in the appendix. The model by Reuvers was meant only as an approximation as long as the polymer solution can be regarded as a semi-infinitely thick medium It is therefore not possible to find composition paths that are valid after the diffusion front has reached the impenetrable support of the solution (e.g the glass plate). After this moment, the composition path must alter; surely the compositions on the interface will change, as shown by Tsay and McHugh [5] They applied an extended model in which the interfacial compositions are allowed to change. Some of their results are however contradictory to expectations (e.g. in their calculations during the delay time, the interfacial polymer concentration decreases in time), and appear to be much more dependent on the input parameters than the Reuvers model. Therefore we may still obtain a general and reliable impression of the diffusional behavior by assuming that even after contact between diffusion front and glass plate, the interfacial compositions still remain approximately the same. In Fig 1 approximate composition paths have been reproduced at four different times after immersion of the supported film in the nonsolvent bath took place The compositions $A$ to $\mathrm{D}$ are the concentrations at the bottom of the film, after diffusion times as indicated in the figure For each curve I means the concentration at the interface between film and bath ( $\mathrm{I}^{\prime}$ is the composition at the polymer film side of the interface, I" at the coagulation bath side; both compositions are connected by a tie-line of the phase diagram) One can see from Fig. 1 that the composition path enters the liquid-liquid demixıng gap in the phase diagram after $21 \mathrm{sec}$ This delay time has indeed been found by turbidity measurements in actual immersion experiments for this system [3c] The fact that we experımentally find the same delay time is somewhat surprising since we considerable oversimplified the model (see above). Durıng the delay time the film looses more solvent to the coagulation bath than it takes up nonsolvent. The concentration profile for the polymer in the film shows a steep gradient at the interface The interfacial layer of increased polymer concentration grows in thickness with the extension of the delay time Once nucleation of the polymer lean phase sets in, startıng somewhere below the interface, there will be an interfacial layer where nucles cannot be formed anymore and a membrane with a dense toplayer is the result Of course for practical membranes one tries to obtain dense toplayers which are as thin as possible.

In Fig. 2 calculated composition paths are reproduced for a system with dioxane as solvent. One sees that even at very short times, part of the compositions in the immersed film are situated in the metastable region of the phase diagram for curves (a) and (b). For these compositions, which are to be found close to the film-bath interface, nucleation of the polymer lean phase will set in immediately after immersion of the film in the coagulation bath. We will see in Part 2 of this series on microstructures in phase inversion membranes that for these instantaneously precipitating films a thin toplayer of a nodular structure is always present. Below this thin layer, nucleation of the polymer lean phase can start. For these cases of instantaneous demixing one also finds the phenomenon of macrovord formation. In the next paragraph a literature survey on macrovoid formation will be given, before presenting the new mechanism on their formation. Two remarks to round off this paragraph on membrane formation background:

- From an evaluation of the origin of the differences in the onset of phase separation in ternary systems [14] it becomes clear that the tendency of mixing of the solvent-nonsolvent pair plays a central role. Instantaneous demixing and macrovords are found for solvent-nonsolvent pairs with a high mutual affinity (high 


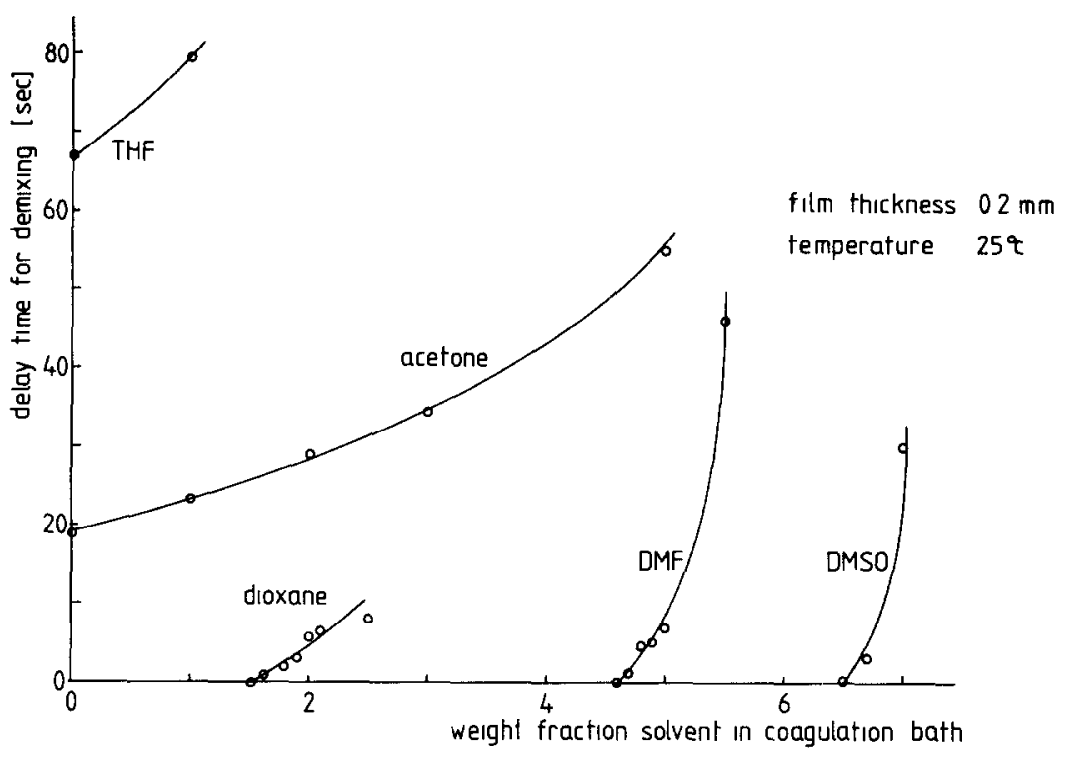

Fig 3 Experimental delay time for the onset of liquid-liquid demıxıng after immersion of a 15 vol \% CA solution, prepared from the indicated solvent, into a coagulation bath with a varying initial solvent-water ratio

positive values of $\mathrm{d} \mu_{\iota} / \mathrm{d} \ln \varphi_{\iota}$ for the binary solution) This is well exemplified by the curves in Fig 3 which show delay times for 15 vol. $\%$ CA solutions in various solvents, immersed in coagulation baths which either consisted of pure water or contained increasing amounts of the solvents indicated in the figure. The delay times were measured by light transmission experıments upon immersion [3c] Going from THF as solvent to DMSO the miscibility with water increases One can see from Fig 3 that the delay time in a pure water coagulation bath decreases going from THF as solvent to acetone In case of dioxane, DMF or DMSO as solvent one has to add increasing amounts of these solvents to the coagulation bath, in order to enforce delayed demixing.

- Other phase separation types than liquidliquid demıxing, 1.e. crystallızation or aggregate formation [15-17] can also occur in polymer solutions. It is clear from kınetic considerations of phase separation [3] that during the relatively fast membrane formation step, nucleation processes which need an ordering pro- cess (such as crystallization or aggregate formation) before taking place will not be operative One can say that membrane morphology is determıned by diffusıon and liquidliquid phase separation In a secondary step formation of fibrillar or other structures in the polymer-ruch phase might occur due to crystallization or aggregation phenomena. This can of course have an effect on the permeability of the dense toplayer

\section{Empirical rules for the occurrence of macrovoids in membranes prepared from ternary systems}

Before we discuss the established theories concerning the initiation and growth of macrovoids we will present some SEM analyses, performed to study the influence of the coagulation bath and casting solution compositions on the formation of macrovoids.

In Fig 4 cross sections are shown of membranes prepared form CA-acetone-water solutions immersed into a pure water bath. The so- 

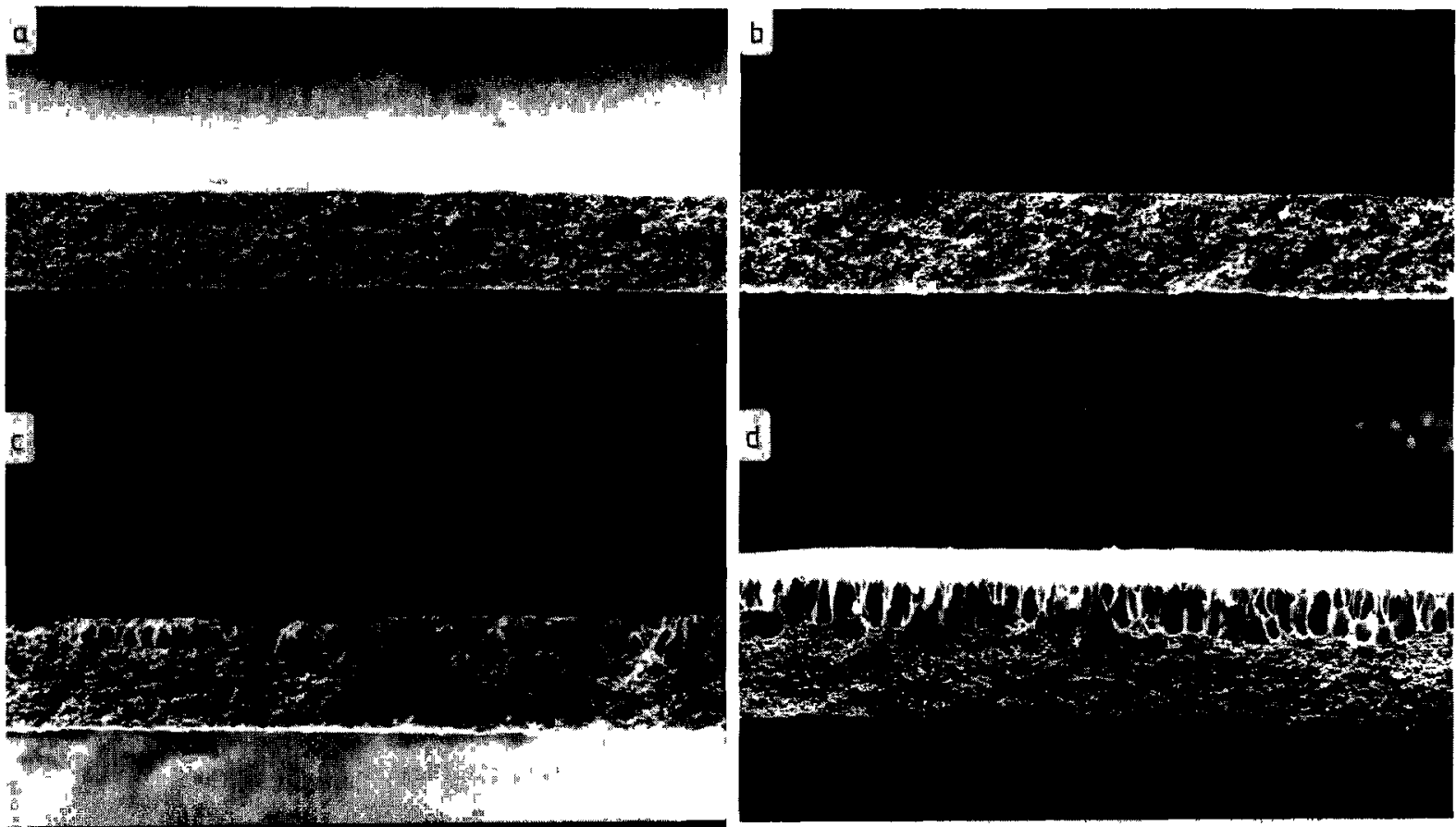

e.
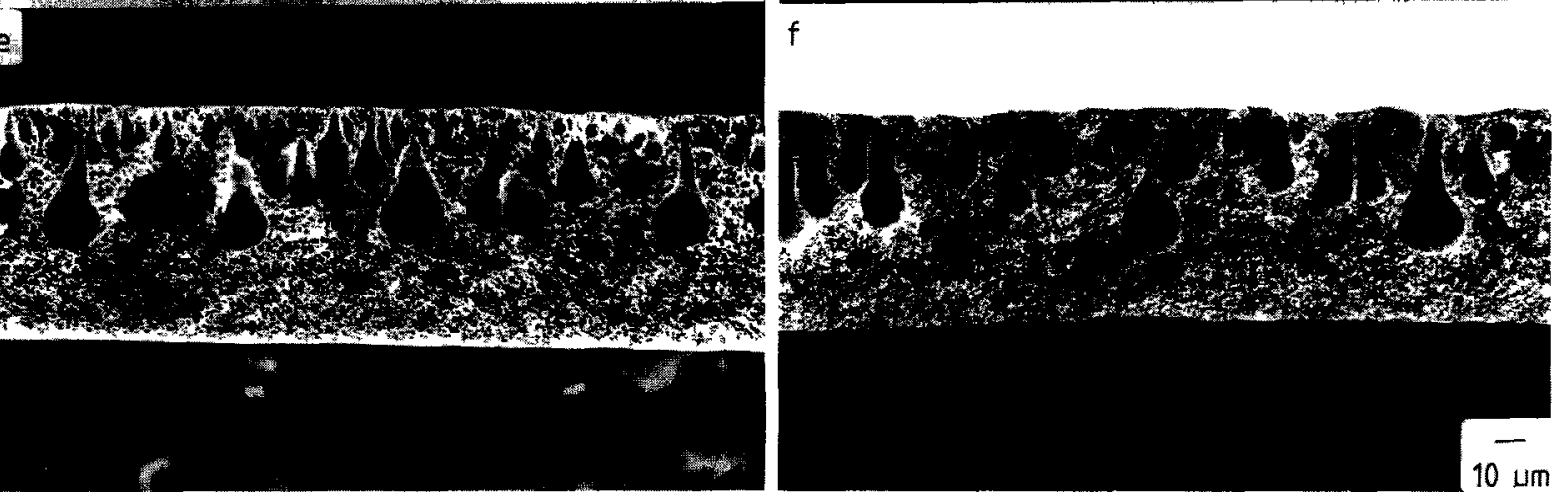

Fig 4 Micrographs of the cross sections of membranes prepared from $200 \mu \mathrm{m}$ thick, $10 \mathrm{vol} \%$ CA solution films, $1 \mathrm{mmersed}$ into a pure water bath, the volume ratio water-acetone in the casting solutions has been varied (a) $0 / 100$, (b) $10 / 90$, (c) $125 / 875$, (d) $15 / 85$, (e) $21 / 79$, (f) $27 / 63$ The enlargement factor is equal for all these micrographs

lution films, initially $200 \mu \mathrm{m}$ thick, consisted of 10 vol.\% CA, dissolved in water-acetone mixtures of varying nonsolvent-solvent ratio It has been shown earlier [ $3 \mathrm{c}$ ] that the transition from delayed to instantaneous onset of liquid-liquid demixing in these immersed films is situated in between a water-acetone volume ratio of $10 / 90$ and $125 / 87.5$ in the casting solution. This turbidimetrically detected transition is accom- panied by a transition for the occurrence of macrovoids, as can be seen from Figs 4(b) and 4(c) Here macrovoids are being formed only in solutions which start to demix instantaneously. By SEM we have also examined the cross sections of some membranes prepared from 15 vol \% CA-dioxane solutions, which were immersed into coagulation baths, with varying water-dıoxane relations Figure 5 shows 


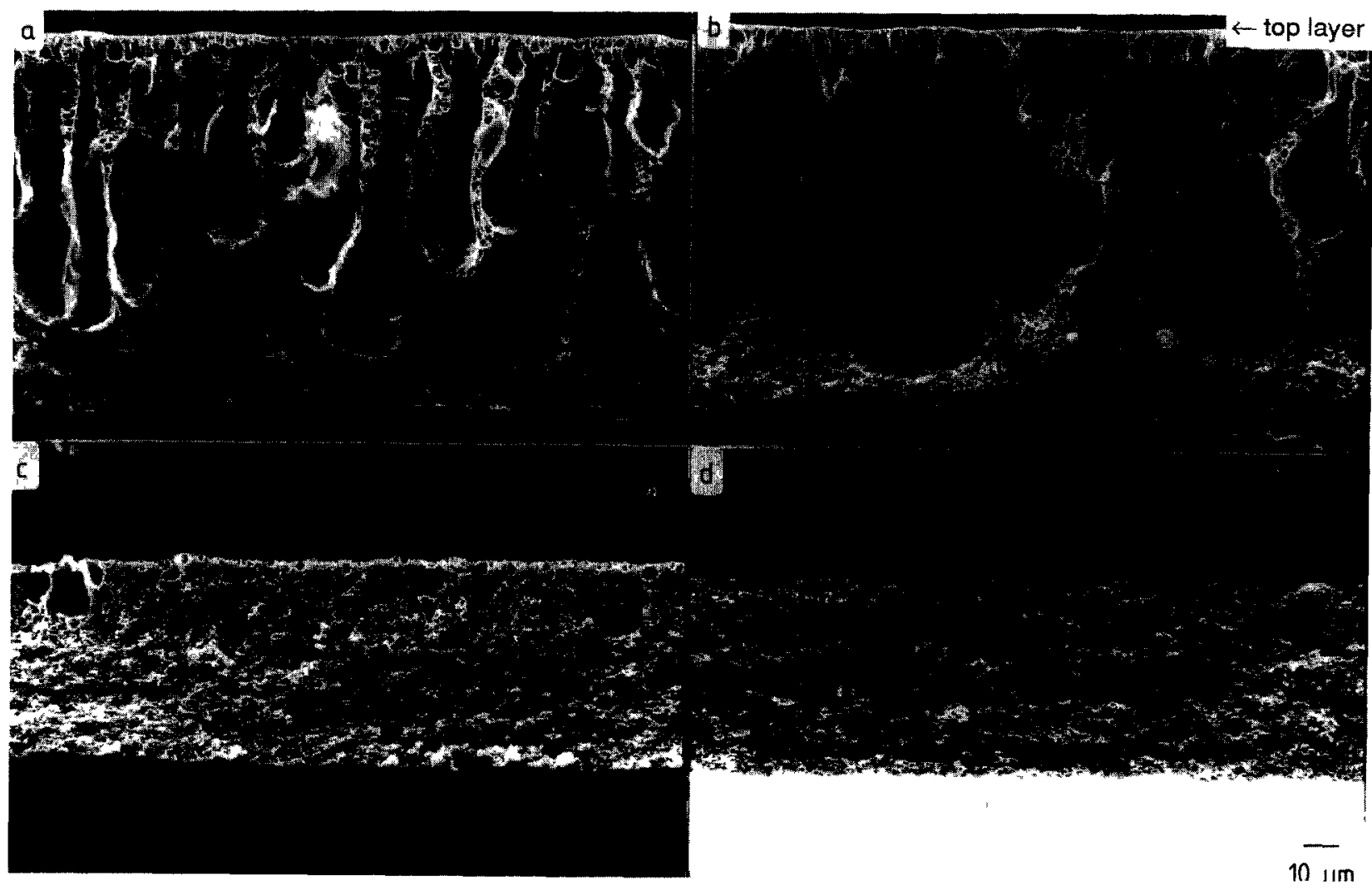

Fig 5 Micrographs of the cross sections of membranes prepared from $200 \mu \mathrm{m}$ thick, $15 \mathrm{vol} \%$ CA-dioxane solution films, immersed into a coagulation bath with a varying dioxane concentration, weight fraction of dioxane in the bath (a) 00 , (b) 01 , (c) 02 , (d) 03 The enlargement factor is equal for all these micrographs

that membranes as obtained in $5(\mathrm{a})$ and $5(\mathrm{~b})$ do contain macrovolds whereas the other ones contain some very small macrovoids $[5(\mathrm{c})]$ or no macrovoids at all $[5(\mathrm{~d})]$.

From light transmission experiments (see Fig 3) it is clear that the change from instantaneous to delayed demixing for $15 \mathrm{vol} \% \mathrm{CA}-$ dioxane solutions) take place at a weight fraction of dioxane in the coagulation bath above 015 Again the occurrence of macrovolds is coupled with the phenomenon of instantaneous demixing These observations are also in accordance with the results of Strathmann et al [8] who performed a similar study on Nomex-DMF (DMAc) casting solutions immersed into various water-DMF (DMAc) co- agulation baths. They observed that instantaneous onset of liquid-liquid demixing 1s accompanied by formation of macrovoids, whereas no marcovolds are formed if liquidliquid demixing starts after a certain minımum delay period

In Fig. 6 cross sections are shown of membranes prepared from various CA-solvent casting solutions immersed into pure water. From these micrographs and the measured periods of delay for the onset of liquid-liquid demixing (Fig. 3) it can be concluded that the transition from instantaneous to delayed onset of demixing obtained by varying the type of solvent, is also accompanied by the disappearance of macrovolds. The same effect can be observed for 

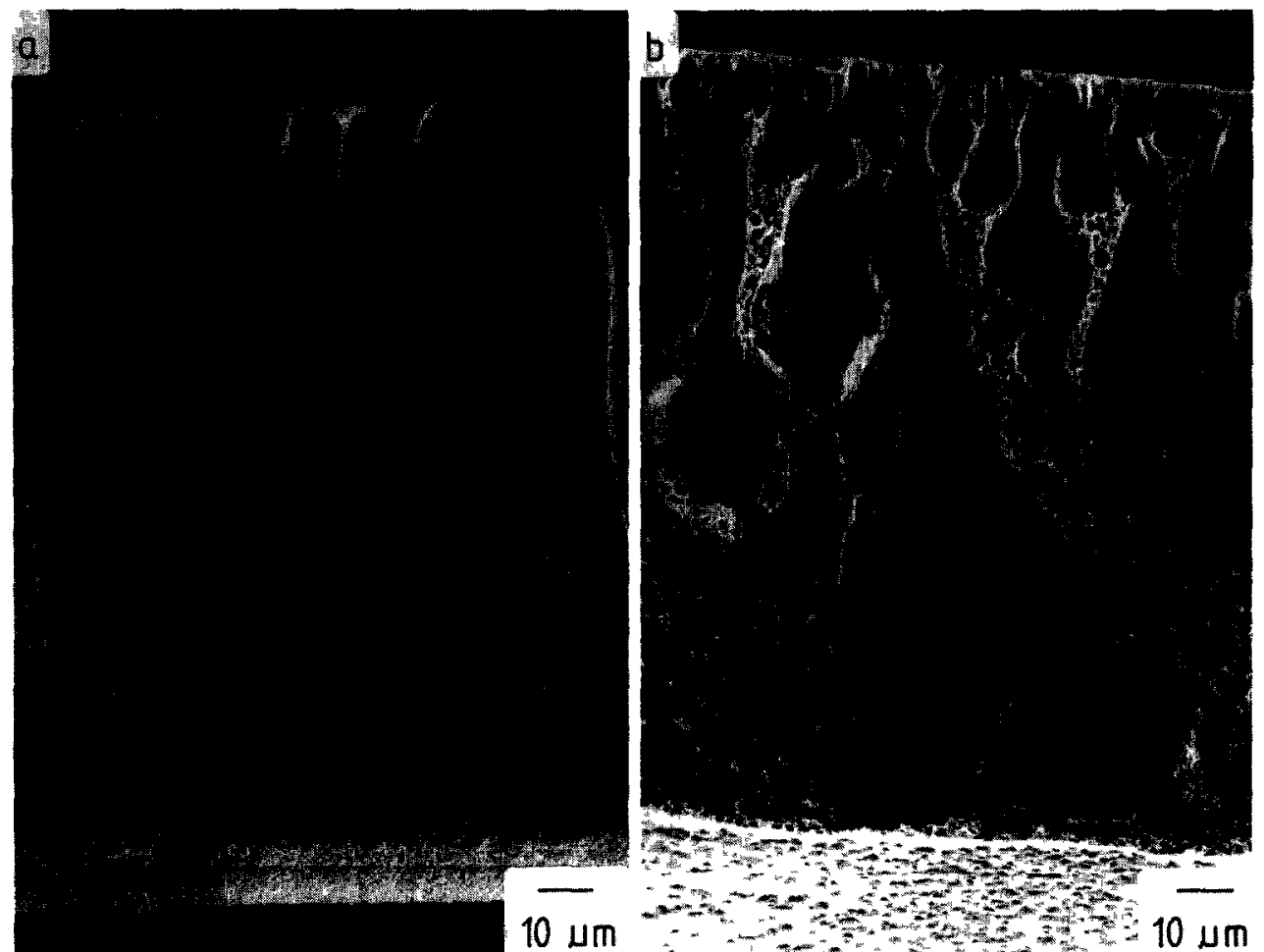

$10 \mathrm{um}$
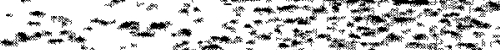

$10 \mathrm{um}$

c

\section{d}

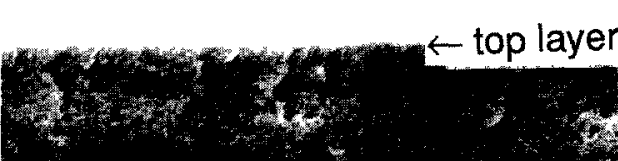
$+3$
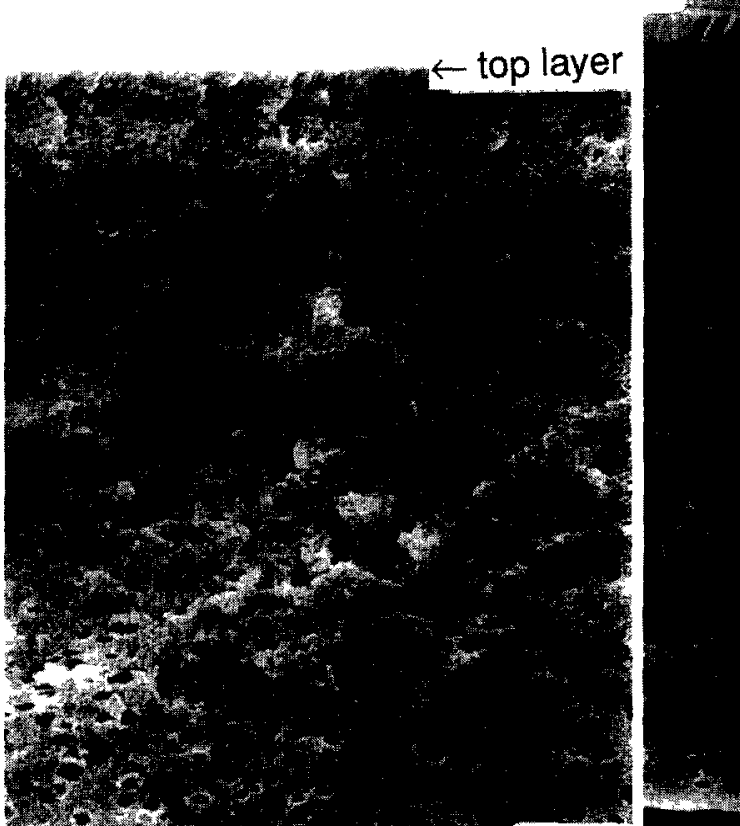
$=x^{2}+2+2$

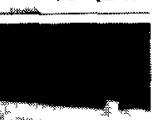

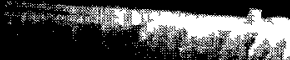

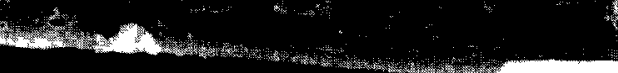


variation of the type of nonsolvent (i.e. i-propanol and ethanol for polysulfone/DMAc casting solutions) [14]. Thus, various examples show that the presence of macrovoids in the ultımate membrane is related with the absence of a delay time for the onset of liquid-liquid demixing upon immersion of the cast solution.

It is possible to avold the formation of macrovords by changing parameters which (probably) do not effect the delay time for the onset of liquid-liquid demixing

- Frommer and Messalam [7] demonstrated that an increase of the initial polymer concentration in a CA-triethyl phosphate casting solution from $7 \mathrm{wt} \%$ to $20 \mathrm{wt} \%$ prohibits the formation of macrovolds upon immersion of the casting solution into an ice-water bath These authors showed that macrovold formation can also be eliminated in immersed CA-DMSO casting solutions, by an increase of the CA concentration from $20 \mathrm{wt} . \%$ to $30 \mathrm{wt} . \%$.

- Strathmann et al. [8] observed macrovord formation to be absent in immersed NomexDMAc casting solutions, upon increasing the Nomex concentration in the casting solution from $20 \mathrm{wt} \%$ to $23 \mathrm{wt} . \%$. It has been stated above that the miscibility of the solvent-nonsolvent combination, used for the preparation of the membrane, has a decisive influence on the type of membrane which will be formed. The solvent-water combinations, with triethyl phosphate, DMSO or DMAc as solvents are excellently miscible systems Therefore, it is very likely that Frommer and Messalam and Strathmann et al provided us with three examples of the absence of macrovoids in membranes, where normally macrovoids should be expected

- Sometimes, it is also possible to avoid the formation of macrovoids in instantaneously de- mixing solutions by the addition of a large amount of nonsolvent to the casting solution This can be concluded from the micrographs in Fig. 7, showing the cross sections of CA membranes prepared from homogeneous CA-dıxane-water casting solutions, immersed into a pure water bath.

With the experimental evidence avalable we would like to propose the following empirical rules for the occurrence of macrovords in membranes prepared from ternary systems:

- membranes without macrovoids are formed in case of delayed demixing, except when the delay time is very short.

- membranes with macrovolds are formed in case of instantaneous demixing, except when the polymer concentration and/or the nonsolvent concentration in the casting solution exceed a minımum value.

\section{Discussion of established theories on the formation of macrovoids}

The formation process of macrovoids can be split up in two parts:

- initiation of macrovoids,

- growth of macrovoids

\section{Initzatıon of macrovolds}

Because the onset of the formation of macrovoids often occurs at the interfacial boundary between the immersed solution film and the coagulation bath, many authors propose that interfacial phenomena cause the initiation of the volds

- Matz [10], Frommer and Messalam [7] and Stevens et al [18] propose that surface tension gradients are the driving forces which account for the initiation of convection cells at the in-

Fig 6 Micrographs of the cross sections of membranes prepared from 15 vol \% CA castıng solutions immersed into a pure water bath Solvents used for the preparation of the casting solution (a) DMF, (b) dioxane, (c) acetone, (d) THF The magnification factor for (a) and (b) differs from that for (c) and (d) 


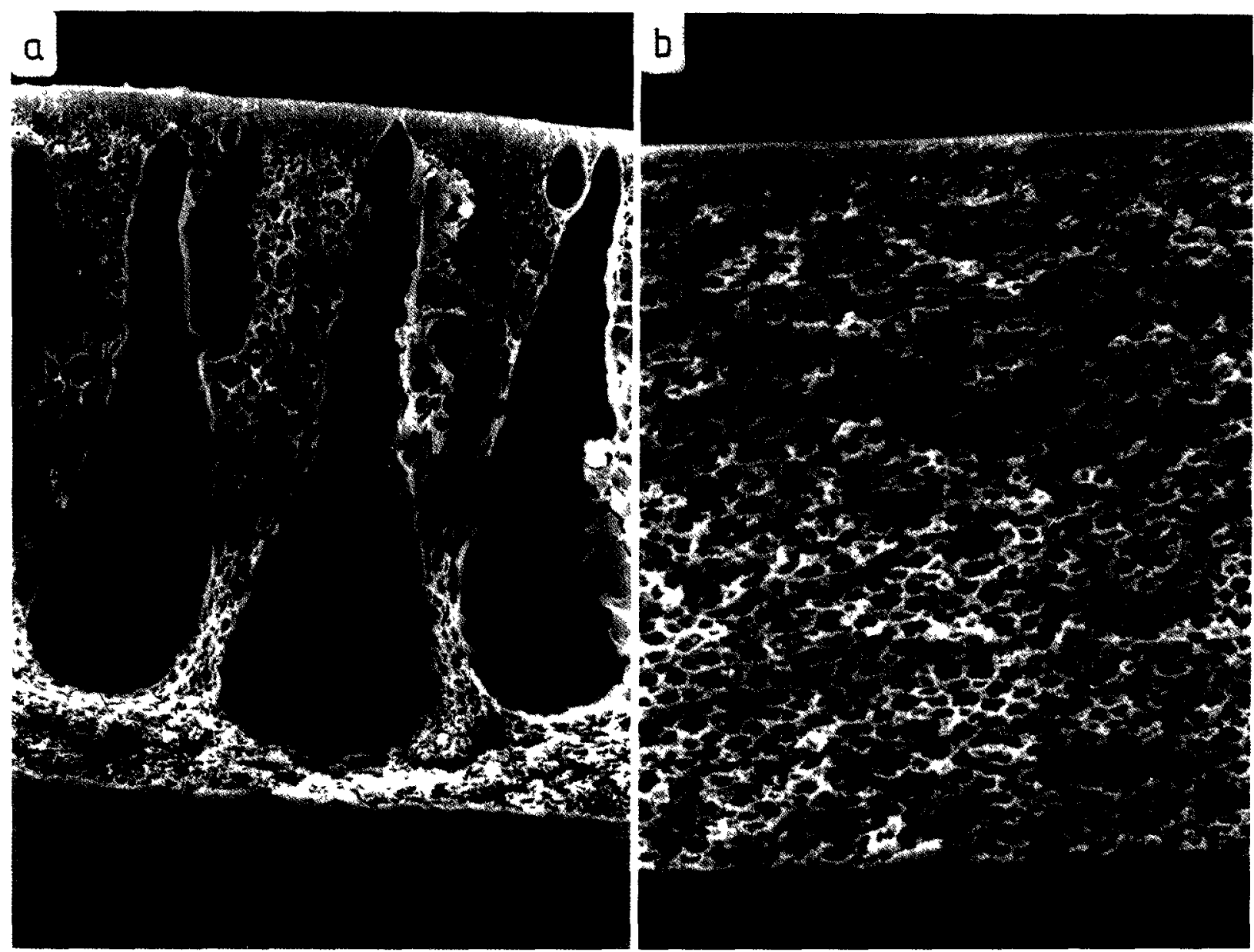

Fig 7 Micrographs of the cross sections of membranes prepared from $200 \mu \mathrm{m}$ thick, $15 \mathrm{vol} \%$ CA-dioxane solution films, immersed into a pure water bath, the volume ratio water-dioxane in the casting solution has been varied (a) $10 / 90$, (b) $25 / 75$ The enlargement factor is equal for both micrographs

terfacial boundary of the film. These authors refer to Sterling and Scriven [19] who showed that periodic convection cells can be generated by interfacial tension variations during the transfer of a solute across a liquid-liquid interface.

- Ray et al [12] proposed that excess intermolecular potential gradients, due to the steep local concentration gradient at the film side of the interfacial boundary, are responsible for the initiation of periodic convection cells

- Strathmann et al. [8] and Graig et al [20] suggested that mechanical stresses cause rupture of the thin toplayer which is formed immediately after immersion of the solution film These rupture points should form the initiation points for the macrovoids

- Broens et al [15] mentioned thinner parts of locally loose arrangement of nodules in the thin toplayer as the initiation points for the macrovoids

We believe that it is not right to attribute the initiation of macrovoids to interfacial phenomena, because it can be observed from cross sec- 
tions of membranes that macrovoid formation sometimes starts at a large distance from the interface between the coagulation bath and the solution film We do agree with Grobe et al [21], who proposed that the nucleation of droplets of the polymer lean phase is respons1ble for the initiation of macrovoids. In the next section we will show that liquid-liquid demixing by means of nucleation of the polymer lean phase can initiate macrovoid formation under circumstances which correspond to the empirical rules for the formation of macrovoids, described in the previous section

\section{Growth of macrovouds}

Different driving forces for the growth of the voids are mentioned in literature.

- Frommer and Messalam [7] suggest that surface tension gradients act as the driving force for the growth of convective cells.

- Strathmann et al [8] propose shrinkage of the matrix in between the growing macrovoids as the driving force

- Smolders [22] suggests that the growth of the vords is caused by solvent, expelled by the surrounding polymer solution, because the polymer molecules change to a less expanded conformation during the increase of the nonsolvent concentration in the immersed polymer solution.

- Grobe et al. [21] and Broens et al [15] propose that a diffusional flow of solvent from the polymer solution surrounding the initiated macrovoid, is responsible for the growth of the voids

The growing macrovold can be regarded as a flow of a polymer lean phase relative to a polymer rich phase From the enumeration given above it can be concluded that some authors suggest that the growing macrovold represents a convective flow, while other authors suggest that the growth is a result of diffusional flows of the various components We will briefly comment on the possibility that the growing macrovords represent convective flows - Convective flows induced by interfacial tension variations [7] Because macrovord formation can start at a large distance from the interface between the coagulation bath and the solution film, in our opinion not only the inıtiation, but also the propagation of macrovoid formation occurs independently from possible interfacial tension variations Even, if interfacial tension variations should induce so called Marangonı convection cells the effects of these cells normal to the interface are nullified by the high viscosity of the solution film according to Sterling and Scriven [19].

- Convectıve flows induced by shrınkage [8] or collapse phenomena [22] In our opınıon shrinkage or collapse phenomena cannot be ruled out beforehand as possible driving forces for macrovoid formation However, it is not evident how the empirical rules for the occurrence of macrovoids in membranes can be explained when shrinkage or collapse phenomena are the driving forces for macrovoid formation.

Matz [10] observed by microscope "considerable convective motion" withın a growing macrovord In our laboratory we have also observed in a precipitating sample between microscope glass plates convective motion of droplets polymer rich phase in a growing macrovold From these types of observations it is sometimes concluded $[7,10]$ that the growing macrovoid itself represents a convective flow. In our opinion this conclusion may not be drawn from these observations. It is also premature to assume that the observed convective motion within the growing macrovold is also present under "normal" circumstances, because this motion can easily be induced by local strong heating effects of the examined sample or preferential wetting of the microscope glass plates. In the next section we will demonstrate that the empirical rules for the occurrence of macrovolds in membranes can be explained if the 
growing macrovold is considered to be a result of diffusional flows

\section{Macrovoid formation by liquid-liquid demixing and diffusional flows}

As has been mentioned above we assume that nucleated droplets of the polymer lean phase in the immersed polymer solution are responsible for the initiation of macrovoids When some of them expand to very large dimensions macrovolds are formed, whereas a sponge-structured sublayer is formed when new nuclei are being generated in front of the existing ones What are the circumstances that determine whether a nucleated droplet will expand freely or not? This question can be answered when we recognize the analogy between: (1) on the one hand the actual situation of a freshly formed nucleus of the diluted phase, separated from the polymer solution in front of it by an interfacial boundary; (ii) on the other hand the undisturbed situation (no nuclei formed yet) of the coagulation bath separated from the freshly immersed polymer solution film by an interfacial boundary. In case (11) the coagulation bath will expand (and the film will shrink) as long as the diffusion process induces a composition path in the film consisting of stable compositions, hence for conditions of delayed onset of demixing. According to the proposed analogy the nucleated droplets of the diluted phase in case (1) will expand when the ternary diffusion process induces stable compositions in front of the freshly formed nuclei (1.e. conditions of delayed onset of demixing) The droplets will not expand further when new nuclei of diluted phase are formed in front of the considered droplets

We shall now examine the change in stability of the solution in front of a layer of nuclel formed under conditions of instantaneous demixing We must realize that the actual solvent/nonsolvent composition in the freshly formed layer of nuclei is shifting to higher and higher solvent concentrations This is caused by the fact that the diffusion front moves further and further away from the interface, and also by the diffusional barriers that are present between the actual droplet-polymer solution interface and the original interface between polymer film and coagulation bath We can draw again the analogy between the mass transfer takıng place to and from the droplet and the polymer solution, and the mass transfer between the total polymer film and the coagulation bath In the latter case (see Fig 2) we see that addition of a certain amount of solvent to the coagulation bath induces a transition from instantaneous (curve a) to delayed demixing (curve c) Since deeper in the polymer solution the solvent concentration at the coagulation front is much higher, we may expect a transition from the instantaneous demixing process taking place globally, to a delay of demıxıng takıng place locally

The general trend of the composition paths in front of the last formed nuclei in the film are described e.g by curve (b) or curve (c) in Fig. 2 , or a curve belonging to still another initial solvent concentration in the coagulation bath If curve (b) approximately describes the compositions in front of the nuclei it can be concluded that new nucle1 will be formed. In case curve (c) describes the composition path, the solution in front of the already formed nucle will remain stable, at least as long as free diffusion occurs The last nuclei formed (or only some of them) will expand because the diffusional flow of solvent from the polymer solution into the nuclei is larger than the flow of nonsolvent from the nuclei into the polymer solution. Hence macrovoids are being formed by a change in precipitation conditions from instantaneous to delayed onset of demixing at a certain distance from the film interface Generally this change of precipitation conditions occurs close to the interface, leaving a rela- 


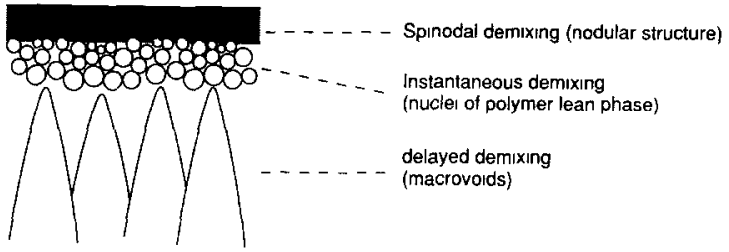

Fig 8 Schematic representation of morphological structures and mechanısms involved in rapidly precipitating solution films

tively thin skın ( $\approx 0,2 \mu \mathrm{m}$ typically) of a different structure This is, however, not always the case. One frequently sees macrovords being formed at distances further away from the membrane toplayer [see e.g Fig 4 (e) and (f)].

Although we do not know the exact diffusional resistance of the toplayer from the first moment of onset of nucleation in the film, we do know that the formation of macrovoids is in many instances accompanied with the occurrence of a skin layer having a nodular structure In Part 2 of this series it will be shown that conditions for spinodal demixing are probable for the interfacial region in rapidly precipitating films The overall picture of structure formation and the mechanısms involved in these films can therefore be schematically represented as in Fig 8

In the next section we will examine the influence of the type of solvent used and of the composition of the casting solution (nonsolvent content, polymer concentration) on the stability conditions of the solutions in front of the nucles formed

\section{Influence of various parameters on the occurrence of macrovoids}

\section{Addition of nonsolvent to the casting solution}

It can be seen from Fig. 4(d) for a 10 vol. $\%$ CA-water-acetone solution (water-acetone ratio: 15/85), and in Fig. 5(a) for a 15 vol.\% $\mathrm{CA}$-dioxane solution, that the nuclei formed beneath the skin layer expand to macrovoids by means of growth (combined with coalescence) Apparently, the created nonsolvent concentrations near the diffusion front were sufficiently low (the solvent concentrations in the droplets sufficiently high) to locally induce delayed onset of liquid-lıquid demixıng in front of already the first formed nucle

It was shown in Fig 2 that addition of solvent to the coagulation bath can yield delay of demixing. In Fig. 9 it is shown that addition of nonsolvent to the polymer solution can give a transition from delayed demixing to instantaneous demixing The delay of demixing is suppressed by addition of nonsolvent to the polymer solution From Figs $4(\mathrm{e})$ and (f) it is indeed clear that addition of extra amounts of water to the $\mathrm{CA}$-acetone-water casting solution suppresses macrovord formation. The occurrence of local delay of demixing is therefore also suppressed by nonsolvent addition.

The same effect of the addition of nonsolvent to the casting solution can be observed for the membranes prepared from CA-dioxane-

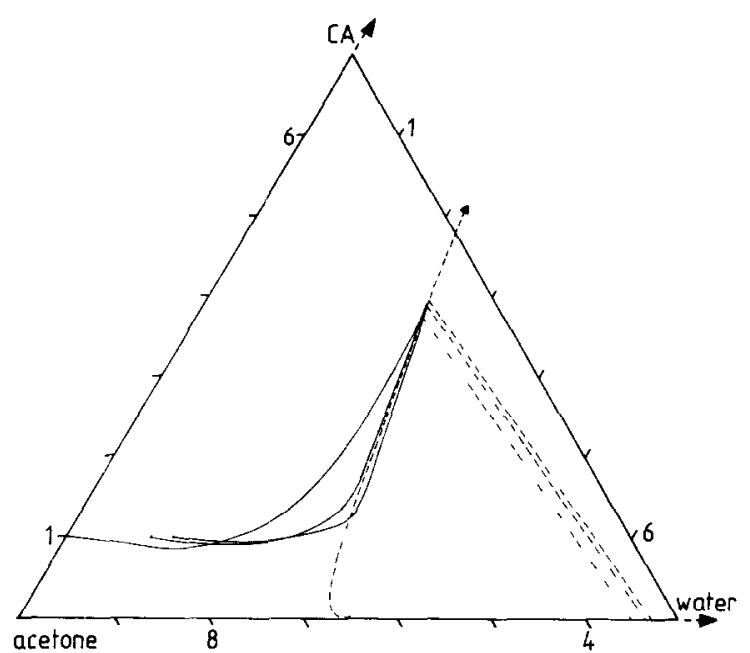

Fig 9 Calculated composition paths for films of $10 \mathrm{vol} \%$ CA solutions immersed into pure water coagulation baths, for different water-acetone ratios in the casting solution (a) $0 / 100$, (b) $10 / 90$, (c) $125 / 875$ 
water solutions [see Figs $7(a)$ and $7(b)$ ]. There is a concentration range, within which irregularities in the nascent skin layer cause an irregular formation of macrovoids. The exact places where macrovoids are formed are determined by irregularities in the diffusion front, caused by inhomogeneities in the skin layer

In Fig 9 we reproduce calculated composition paths for films of $10 \mathrm{vol} \% \mathrm{CA}$ solutions immersed into pure water, but for different water-acetone ratios in the casting solution (a) $0 / 100$, (b) $10 / 90$ and (c) $12,5 / 87,5$ volume ratios water-acetone [3c] As already discussed earlier, we see that the composition paths shift closer to the binodal region and even across the binodal at a certain nonsolvent content of the casting solution. We also see that the polymer concentration in the interfacial region slightly increases with increasing nonsolvent concentration

It can easily be deduced that a higher solvent concentration is necessary in the coagulation bath, to obtain delayed onset of liquid-liquid demixing in an immersed film of which the nonsolvent concentration has been increased According to our model the elimination of macrovoids shown in the Figs 4 (e), 4(f) and 7(b) can be explained by a suppression of local delay of demixing, upon addition of a certain minimum amount of nonsolvent to the casting solution.

Variation of the polymer concentration in the casting solution

Now we want to known in which way the process of macrovoid formation is affected by an increasing polymer concentration in the casting solution. Let us consider CA-dioxane casting solutions immersed into a pure water bath. From Fig 7 (a) it can be seen that in the immersed 15 vol.\% CA solution macrovoid formation occurs Upon increasing the polymer concentration of the casting solution two counter active effects can be notıced:

(1) Figure 10, representing calculated composition paths at two different CA-dioxane concentrations, shows that with increasing polymer concentration in the casting solution, the interfacial polymer concentration strongly increases Since this will hinder (slow down) the indiffusion of nonsolvent, this would promote the formation of macrovords according to our model

(11) On the other hand turbidity data on precipitating CA-dioxane films with increasing CA concentrations [23] show that the minimum dioxane content in the coagulation bath to enforce delayed demixing also increases strongly with the polymer concentration Therefore the enrichment of solvent content in the nucles of the polymer lean phase upon precipitation might have so high a threshold value that it will not be reached at all This then means that conditions of delayed demixing are not realızed and hence no macrovoids will be formed. It is impossible to exactly predict by calculation at which polymer concentration macrovoid formation will stop in the CA-dioxane casting solution

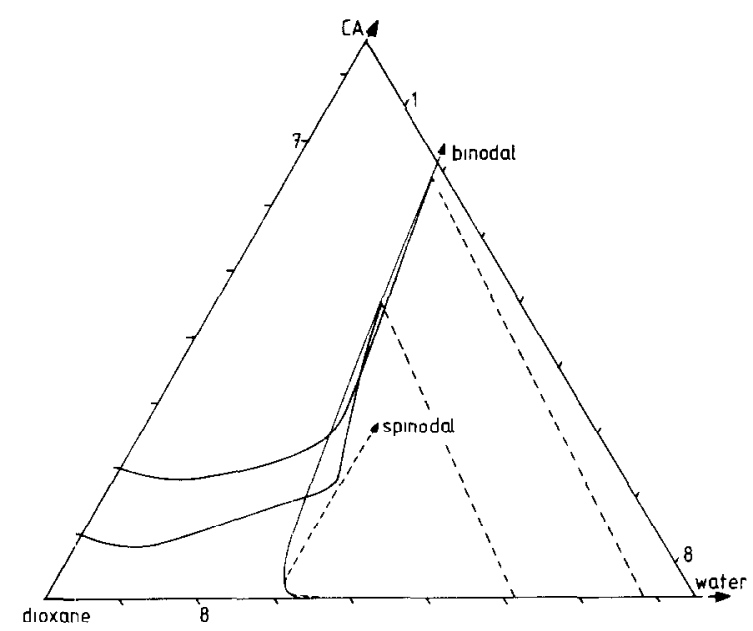

Fig 10 Calculated initial composition paths for CA-dioxane solutions with varying CA concentration, immersed into a coagulation bath consisting of pure water 


\section{Varration of the type of solvent used}

If dioxane is exchanged by a solvent that mixes better with water, the surface composition of the immersed film approaches the composition of water swollen CA already at a relative low polymer concentration in the casting solution This means that the resistance of the freshly formed skin layer can hardly increase any further upon raising the polymer concentration in the casting solution. Thus the absence of macrovolds in immersed CA-DMSO or CA-triethyl phosphate casting solutions, upon increasing the polymer concentration in the casting solution (observed by Frommer and Messalam [7]) can be explained in our model by the fact that the minımum amount of solvent in the coagulation bath, necessary to obtain delayed onset of demixing, increases with increasing polymer concentration in the casting solution

More experiments should be performed to further test our model:

- It has to be examined at which polymer concentration in the casting solution macrovoid formation stops to take place. This must be examined for good and poor miscibility of solvent-nonsolvent combinations

- For the same solvent-nonsolvent combinations, light transmission measurements have to be performed in order to obtain experimental values for the minimum amount of solvent in the coagulation bath, necessary to obtain delayed onset of demixing in casting solutions with a varying polymer concentration.

- The initial rate of growth of macrovords must be compared with the rate of movement of the interface between the immersed polymer solution and the coagulation bath, for a solvent concentration in the coagulation bath which is high enough to prevent instantaneous onset of liquid-liquid demixing

\section{Conclusions}

The occurrence of macrovolds in membranes prepared from ternary systems can be predicted with the following empirical rules

- Membranes without macrovoids are formed in case of delayed demixing, except when the delay tıme is very short

- Membranes with macrovords are formed in case of instantaneous demixing, except when the polymer concentration and/or the nonsolvent concentration in the casting solution exceed a minımum value

According to the model proposed:

- Macrovoids are formed from freshly formed nucles of the diluted phase if the composition in front of the nuclei remains stable for a relatively long period;

- Macrovoids expand as a result of diffusional flows

- Macrovoids are formed just beneath the skın layer of the solvent concentration in the freshly nucleı exceeds a certain minımum value This conclusion is contrary to the widely proposed hypothesis according to which macrovolds are formed at certain weak spots in the nascent skin layer.

- Macrovoid formation is not opposed by an increase of the viscosity of the cast solution (as is generally assumed), but by an increase of the minimum (threshold) solvent concentration in the layer of freshly formed nuclei, necessary to obtain delayed onset of liquid-liquid demixing

\section{References}

1 C Cohen, G B Tanny and S Prager, Diffusion-controlled formation of porous structures in ternary polymer systems, J Polym Scı, 17 (1979) 477

2 L Yilmaz and A J McHugh, Modelling of asymmetric membrane formation I Critıque of evaporation models and development of a diffusion equation formalısm for the quench period, J Membrane Sc1, 28 (1986) 287 
3 (a) A J Reuvers, Membrane Formation, Diffusion induced demixing processes in ternary polymeric systems, Dissertation Univ of Twente, December 1986 (b) A J Reuvers, JW A van den Berg and C A Smolders, Formation of membranes by means of $1 \mathrm{~m}$ mersion precipitation Part I A model to describe transfer during immersion precipitation, J Membrane Sc1 , 34 (1987) 45

(c) A J Reuvers and C A Smolders, Formation of membranes by means of immersion precipitation Part II The mechanism of formation of membranes prepared from the system cellulose acetate-acetonewater, J Membrane Scı , 34 (1987) 67

4 R M Boom, I M Wienk, Th van den Boomgaard and C A Smolders, Microstructures in phase inversion membranes Part 2 The role of a polymeric additive, J Membrane Sc1, 74 (1992) 277

5 C S Tsay and A J McHugh, Mass transfer modellıng of asymmetric membrane formation by phase inversion, J Polym Scı , Polym Phys, 28 (1990) 1327

6 M A Frommer and D Lancet, The mechanism of membrane formation Membrane structures and their relation to preparation conditions, in $\mathrm{H} \mathrm{K}$ Lonsdale and $\mathrm{H} \mathrm{E}$ Podall (Eds), Reverse Osmosis Membrane Research, Plenum Press, New York, NY, 1972, p 85

7 M A Frommer and R M Messalam, Mechanism of membrane formation VI Convective flows and large void formation during membrane precipitation, Ind Eng Chem, Prod Res Dev, 12 (1973) 328

8 H Strathmann, K Kock, P Amar and R W Baker, The formation mechanism of asymmetric membranes, Desalınation, 16 (1975) 179

9 V Grobe and G Mann, Strukturbildung beim spinnen vom Polyacrylnitrillosungen in wasserige Fallbader, Faserforschung Textlltechn , 19 (1968) 49

$10 \mathrm{R} \mathrm{Matz}$, The structure of cellulose acetate membranes I The development of porous structures in anisotropic membranes, Desalination, 10 (1972) 1

11 I Cabasso, E Klein and J K Smith, Polysulfone hollow fibers II Morphology, J Appl Polym Scı, 21 (1977) 165
12 R J Ray, W B Kranz and R L Sanı, Linear stability theory model for finger formation is asymmetric membranes, J Membrane Sc1 , 23 (1985) 155

13 F Altena and C A Smolders, Calculation of liquidhquid phase separation in a ternary system of a polymer in a mixture of a solvent and a nonsolvent, $\mathrm{Ma}-$ cromolecules, 15 (1982) 1491

14 A J Reuvers, Dissertation Unıv of Twente 1986, Chap 7, Control of membrane morphology

15 L Broens, F W Altena and C A Smolders, Asymmetric membrane structures as a result of phase separation phenomena, Desalination, 32 (1980) 33

16 J G Wumans, H J J Rutten and C A Smolders, Phase separation phenomena in solutions of poly $\left(2,6-d_{1}\right.$ methylphenyleneoxide) in mixtures of trichloroethylene, 1-octanol and methanol relationship to membrane formation, J Polym Sc1, Polym Phys Ed, 23 (1985) 1941

17 A Labudzınska and A Ziabıcki, Effect of compos1tion and gelation conditions on structural changes accompanying the gelation of PAN, PVA and gelatin solutions, Kolloid Z Z Polym , 243 (1971) 21

18 W E Stevens, C S Dunn and C A Petty, Surface tension induced cavitation in polymeric membranes during gelation, Paper presented at 73rd AIChE Annual Meeting, Chicago, ILL, 1980

19 V Sterling and L E Scriven, Interfacial turbulence Hydrodynamic instability and the Marangoni effect, AIChE J , 5 (1959) 514

20 J P Craig, J P Knudsen and V F Holland, Characterization of acrylıc fiber structure, Textıle Res J , 32 (1962) 435

21 V Grobe, G Mann and G Duwe, Ausbildung von Strukturen bei der Koagulation von Polyacrylnitrillosungen, Fasenforsch Textlitechn , 17 (1966) 142

22 C A Smolders, Morphology of skinned membranes A rationale from phase separation phenomena, in A R Cooper (Ed), Ultrafiltration Membranes and Apphcations, Plenum Press, New York, NY, 1980, p 161

23 A J Reuvers, The connection between non-1deality parameters of ternary systems and the mechanism of membrane formation by means of immersion precipitation, Dissertation Univ of Twente 1986, Chap 6 


\section{Appendix}

Parameters used in Figs 1 and 2, in combınation with the equations derived in Refs. [3].

Thermodynamic parameters

Material properties

\begin{tabular}{lcl}
\hline Material & $\begin{array}{l}\text { Mol weight } \\
(\mathrm{g} / \mathrm{mole})\end{array}$ & $\begin{array}{l}\text { Specific volume } \\
\left(10^{-3} \mathrm{~m}^{3} / \mathrm{kg}\right)\end{array}$ \\
\hline $\mathrm{CA}$ & 27,000 & 0.717 \\
Acetone & 58.0 & 07 \\
Dioxane & 88.12 & 0.967 \\
Water & 18.02 & 1.003 \\
\hline
\end{tabular}

Interaction parameters

\begin{tabular}{lll}
\hline Pair & CA-acetone-water & CA-dioxane-water \\
\hline$g_{12}:$ & $0.979+1.127 \exp \left(-2306 \varphi_{1}\right)$ & $1.389-0607 \varphi_{1}+1050 \exp \left(-4731 \varphi_{1}\right)$ \\
& $+0.292 \exp \left(-12.564 \varphi_{1}\right)$ & $+0.50 \exp \left(-19.922 \varphi_{1}\right)$ \\
$g_{23} \cdot$ & $0.645-0.11 \varphi_{2} /\left(\varphi_{3}+\varphi_{2}\right)$ & $0.62-011 \varphi_{2} /\left(\varphi_{3}+\varphi_{2}\right)$ \\
$g_{13}:$ & 14 & 14 \\
\hline
\end{tabular}

Kinetıc parameters

\begin{tabular}{lll}
\hline Parameter & CA-acetone-water & CA-dioxane-water \\
\hline$\frac{\bar{v}_{1} R T}{M_{2} R_{12}}\left(10^{-9} \mathrm{~m}^{2}-\mathrm{sec}^{-1}\right)$ & 1.25 & $1-025 \varphi_{2}$ \\
$\frac{\bar{v}_{3} R T}{M_{2} R_{23}}\left(10^{-9} \mathrm{~m}^{2}-\mathrm{sec}^{-1}\right)$ & $1.66 \times 10^{1-517 \varphi_{3}}$ & $2.73 \times 10^{-517 \varphi_{3}}$ \\
$\frac{\bar{v}_{2} R_{13}}{\bar{V}_{1} R_{23}}$ & 05 & $10^{-\varphi_{3}}$ \\
\hline
\end{tabular}

